\title{
METHODS OF STUDYING FIELD GERMINATION AND SEEDLING PHYSIOLOGY: PRESENT POTENTIAL AND DRAWBACKS
}

\author{
HANNE N. RASMUSSEN
}

Forest \& Landscape, LIFE faculty, University of Copenhagen, Rolighedsvej 32, DK-1958 Frederiksberg C, Denmark. E-mail: HNR@life.ku.dk; Tel.: +45 35331703; Fax: +45 35331508

\begin{abstract}
Seed germination and seedling growth are equally important processes for population survival. Observation of germinating orchid seeds in a natural substrate is inhibited by their minute size. There are basically two approaches to studying germination in situ: Search for spontaneous seedlings, and field sowing experiments; these may be supplemented with experiments in vitro. Finds of spontaneous seedlings are clearly irregular and fortuitous but may yield valuable, if fragmentary, input to hypothesis building. An understanding of the habitat dynamics may be helpful in the search of such seedlings. The seed package technique for germination in situ and its various adaptations can yield considerable information concerning spatial distribution and timing, identity of participating fungi, seedling growth rate and stimulatory environmental conditions. This approach constitutes the backbone to studies of germination ecology and still holds much unexplored potential. However, conditions that are suitable for orchid seed germination in nature are not necessarily identical to those supporting adult plant growth. Experiments in vitro on artificial substrate normally employs either immature or strongly surface sterilized seeds, and thus the results can be misleading regarding the behavior of natural seeds and seedlings in the field. However, such experiments may be designed for added information on specific questions, provided that growth conditions are manipulated suitably. Experiments in vitro, especially symbiotic and on series of minimal media, have much to offer in supporting and extending field observations of substrate and site preferences.
\end{abstract}

Keywords: seedling recruitment, dust seeds, orchids, species conservation

\section{Introduction}

With the ultimate goal of turning dwindling orchid populations into prolific ones, there is a need to understand how seedling recruitment takes place in natural habitats and to identify factors that favor seedling recruitment.

The minute orchid seeds are often thought to be practically unlimited in their dispersal. However, the statistical chance of seed rain clearly decreases dramatically with the distance to the mother plant. For instance, in a species of Cephalanthera, Chung et al. (2004) found a genetic structure consistent with a dispersal of less than $10 \mathrm{~m}$ for the majority of seeds. Impressing reports of long distance dispersal over more than 10-15 km (Ridley 1930; Crackles 1975; Willems 1982) need to be regarded as extremely rare cases. Orchid seeds are also often claimed to lack reserve nutrients. However, embryo cells of species that have been studied are literally packed with the most concentrated forms of seed reserves: lipids and protein, occasionally some additional starch. Though the amount is obviously limited by the seed size, these reserves are mobilized during germination just as in any other seed (e.g., Manning and Van Staden 1987; Rasmussen 1990; Richardson et al. 1992).

Recent evidence indicates longevity of orchids seeds in the wild, in spite of seemingly fragile seed coats. Based on sowing experiments, survival of terrestrial orchid seeds for 5-10 years in the ground is plausible, at least for some species (Whigham et al. 2006). Seed packets extracted from the ground after a number of years may contain ungerminated seed that are still viable. It seems possible that the seed reserves are involved in the extension of seed life and that longevity could thus be related to specific seed size. If so, we might expect seeds of epiphytic species to be generally more short-lived as seed size tends to be smaller than terrestrial species (Rasmussen 1995). The consequences of a potentially large seed bank will be that fluctuations in seed set from year to year is damped, and seed resources in ailing populations with low fruiting success may not be so small as they would seem. The more important limitation to seedling recruitment could thus be unfulfilled germination requirements.

\section{Methods}

The scope of this review is to outline some of the potential and pitfalls in various approaches to the study of orchid germination in situ, from a personal experience and perspective. The review is not intended to give an exhaustive treatment of the published data in the field.

\section{Germination in situ - spontaneous or arranged}

In essence there are two kinds of in situ germination: the spontaneous seedling originating from a naturally dispersed seed, and the manipulated kinds that result from some kind of sowing technique in or on the natu- 
ral substrate. The first kind lends itself only to post-hoc analysis and hypothesis building, whereas the latter kind has much potential for experiments.

\section{Spontaneous seedlings}

The statistical chance of finding seedlings is normally largest in proximity to the mother plant (Diez 2007; Jacquemyn et al. 2011). However, it is important to realize that optimum conditions for germination are not necessarily identical to optimum conditions for seedling growth, which in turn may not be identical to optimum conditions for adult plant. Many orchids live in successional habitats and are long-lived so that they may exploit or adjust to a gradual change of scenery. This is exemplified in the North American Tipularia discolor whose seedlings were invariably found on rotting logs (of diverse wood species, mainly angiosperm, Table 1 in Rasmussen and Whigham 1998b). When the first leaves appeared above the substrate, the wood was already soft and in brown decay, and when the plant reached flowering stage some years later, the logs would have disintegrated and melted into the forest floor (Rasmussen and Whigham 1998b). That kind of habitat dynamics needs to be taken into consideration in searches for spontaneous seedlings.

Having identified a promising locality with an adequate density of flowering and fruiting plants there is a need to attend to season. A simple fact such as germination season is unknown for the majority of orchid species. Some species may develop so quickly that seedlings after a short while are no longer distinguishable from the more established plants. In a population of long-lived adults and seed longevity there is no absolute need for germination each year. Germination may fail when climatic conditions do not fulfill the requirements of either freshly dispersed seeds or seeds stored in the "bank". Terrestrial seedlings, when they develop their first foliage, tend to have a shorter foliage season than the adult individuals (pers. obs.). The reason could be that seedlings still have poorly developed roots, and a predominantly mycophagous lifestyle. These seedling leaves may escape attention as they are withered at the same time that adult plants are flowering. Weather conditions are equally important in the hunt for spontaneous terrestrial seedlings, as a loose
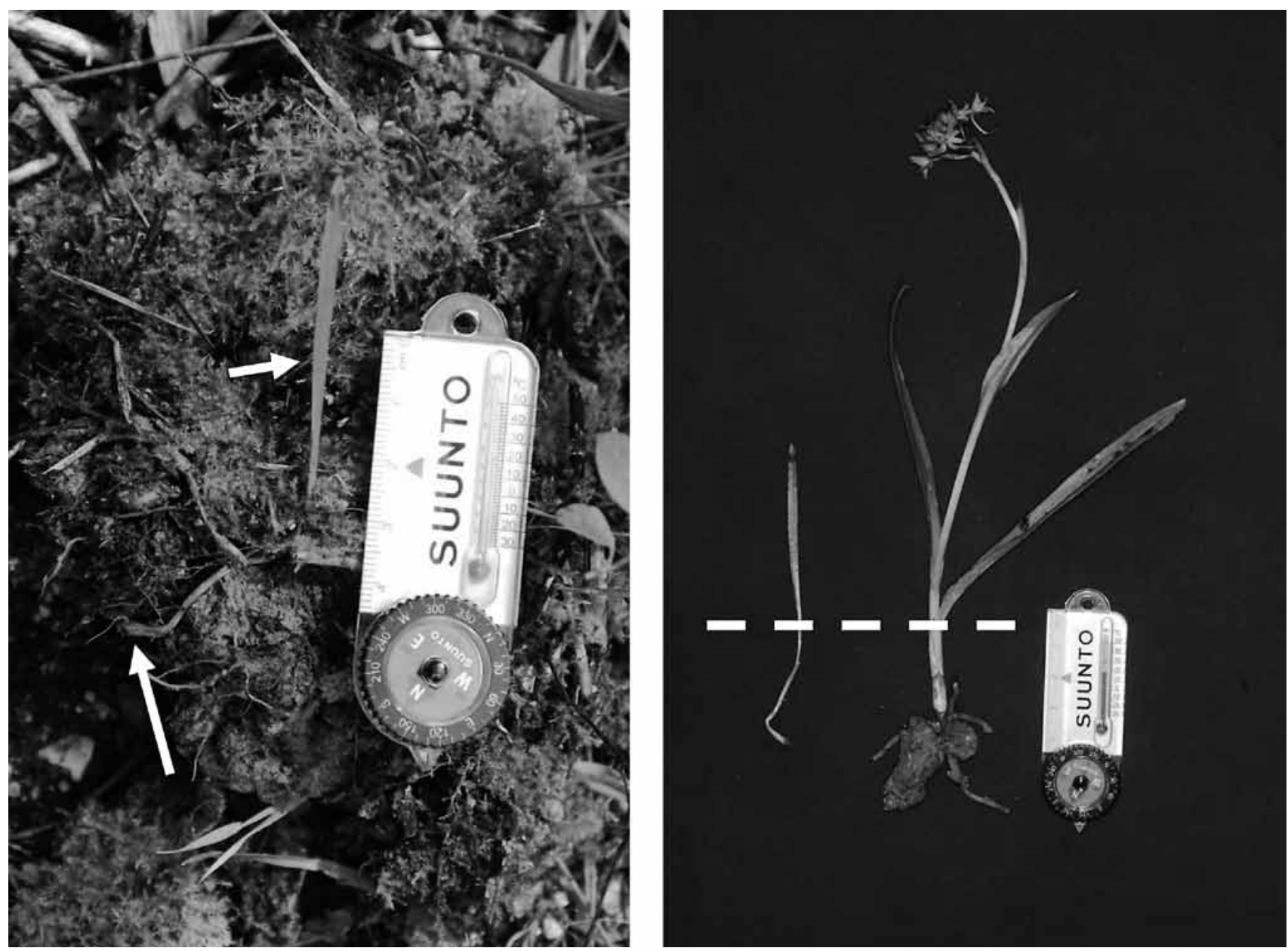

Fig. 1 Spontaneous seedling of Anacamptis pyramidalis in situ. a. It takes some patience and experience to find young seedlings. At best they should be younger than this one whose first seedling leaf is showing above ground (short arrow). The protocorm is however still intact in the ground (long arrow). Finds such as these yield information about the typical location, substrate conditions and depth of germination, the latter seen in (b) in comparison to depth of a flowering plant. Stippled line indicates approximate ground level. Scale: $5 \mathrm{~cm}$, photos Finn N. Rasmussen. 
granular and not too dry soil increases the chance of detecting them. In a subtropical climate, the clayey calcareous soil is most workable after heavy rains.

Most terrestrial seedlings have been found at a small depth in shallow soils (references in Rasmussen 1995) but that could be a convenience bias. If the adults are deep set, so most likely are the seedlings (Fig. 1). In this case the seeds will have sifted through the soil to a depth of approximately $50 \mathrm{~mm}$, which in the Mediterranean climate might adequately protect the seedling against critical water stress during the dry season. Often when a seedling is found, there is probably several close by in different growth stages, indicating the location of a preferred germination patch. Genetic analysis of plant patches indicates that also epiphytic orchids tend to germinate in clusters (Trapnell et al. 2004). Unfortunately, without a means of age determination, it is uncertain whether such patches arise from a single colonization by one cohort of seeds, or repeated germination events over several seasons.

One or more spontaneous seedlings give a few study options. The seedling may be structurally described, and often the specific identify of the seedling in question also needs to be ascertained. The microsite in which it germinated some years previously can be chemically and physically characterized. Isolation of the symbiont fungus is an obvious choice, preferably from still leafless seedlings because they have most likely retained the same fungal strain that stimulated germination. A mycelium growing out from a peloton taken from the seedling, or just the peloton itself, will enable a safer identification than a general DNA analysis of the fungal flora on and in the seedling. Provided the fungus in question is culturable, a pure culture may be used for testing physiological requirements in vitro on a series of minimal media, and reinfection of seeds in vitro could be carried out to support the evidence of a symbiotic relationship. Unfortunately the fungi are not always easily cultivated and more sophisticated methods such as co-culture with a living host tree may be required (McKendrick et al. 2000).

From a population point of view the evidence gained from spontaneous seedlings is clearly very fragmentary unless the search were carried out in a well defined pattern that could tell us about density and size distribution of the underground stages. Even provided such systematic seaches could be justified, the one-time destructive approach leaves the age structure and seasonality unknown. In spite of such limitation, Bernard's (1899) chance encounter of seedlings of Neottia nidus-avis was indeed decisive for leading him to describe and interpret symbiotic orchid germination in the first place - a description that largely retains it validity today.

\section{Sowing in situ}

Field sowing experiments are a very different approach which has been in use for nearly two decades now (Rasmussen and Whigham 1993) and used extensively for terrestrial species. The main problem of retrieving the tiny orchid seeds in soil has been solved with seed packets of various construction, but methods for sowing of epiphytes are lagging behind (see however, Corey et al., this volume). A problem of seed packets is that the number of minute seeds cannot be precisely regulated and are not quantified initially, so at later retrievals a seed mortality rate cannot be ascertained.

Field sowings could be a follow-up on spontaneous finds, by which one has formed hypotheses about the time, and conditions that favour germination. Seed packets can be experimentally inoculated with cultures of from previously identified symbionts, or used for symbiont trapping (e.g., Brundrett et al. 2003; Keel et al. 2011). Most evidence that we have so far gained from seed packet experiments has been about symbiont preferences (e.g., Swarts et al. 2010), timing of germination and seed longevity. Seed packets have also been used for testing spatial distribution and distance effects from mother plants (e.g., Batty et al. 2001), from mycelia and from symbiont host tree in cases when the fungus involved is ectomycorrhizal (McKendrick et al. 2000). Seed packets also lend themselves to testing for substrate chemical and physical quality. This can be accomplished by placing test plots along a gradient, e.g. according to humidity or exposure. Another option is to combine sowing with manipulated soil quality, for instance by adding various kinds of humus (Whigham et al. 2002). In general one may say that much more needs to be done to yield a more complete picture of field seed behavior in the vast orchid family. Approaches used in the above-mentioned and similar works are far from being fully developed and explored.

One of the advantages of field sowings is that one can use natural and untreated seeds and plant them at the time of year when they are naturally dispersed. Large experiments enable us to sample repeatedly over a long time so that the timing, season of germination and speed of seedling growth can be estimated. The seeds of one packet belong in the same cohort and thus the natural variation in seedling development may be estimated. Uncertainty as to time frame of the experiment and the replicate requirement is a problem, if the seed dormancy behavior of the orchid species in quesiton is unknown. However, there is evidence that an inspect-and-replace procedure can be carried out without losing seed viability and so there is an option for extending a long term experiment somewhat if required (Rasmussen and Pedersen, this volume).

Seed packets usually let in microorganisms and small fauna that might play a role either in preparing the seeds for germination or destroying them. If larger animals are involved in promoting germination, for instance earthworms, it is a drawback that seed packets shut them out. The impact of soil organisms could be investigated by employing varying mesh sizes for seed packets. Another drawback to seed packets is that the seeds are prevented from sifting through to deeper soil layers. 


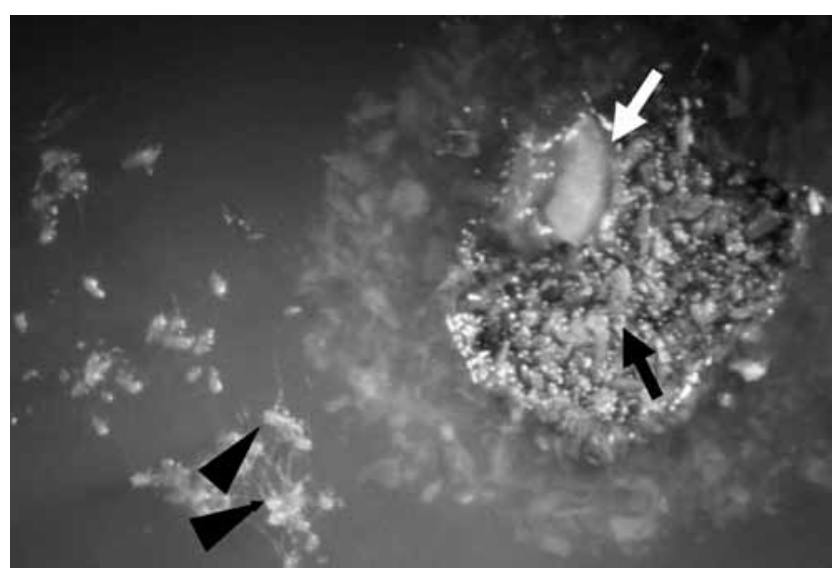

Fig. 2 Near-natural substrate formulations used in vitro yield information about the requirements of seeds and seedlings, e.g., this experiment to explore preferences for certain types and stages of decomposing wood. Water agar supplemented only with sterilized ground rotting wood (black arrow) supports the development of a mycelium from a pure culture (inoculate at white arrow). Germinating seeds and symbiotic seedlings (arrowheads) located nearby. Photo: Hanne N. Rasmussen.

\section{Experiments in vitro}

Compared to spontaneous seedlings, field sowing techniques may further qualify our hypotheses about which conditions favour germination and seedling recruitment. To this we can add yet another approach, in vitro cultures. All too often orchids are germinated on artificial substrates with numerous ingredients and several ill-defined additives that they would not encounter in nature. This makes sense if the aim is simply to produce some plants but it has of course very little bearing on what goes on in situ. Much information can however be gained by simplifying the substrate and/or including a mycobiont (e.g. Rasmussen and Whigham 1998a). Based on hypotheses as to the natural subtrate we could test a variety of natural sources of humus (Fig. 2), competing mycobionts in co-culture (McCormick et al. 2006), and physiological requirements of the symbiotic seedlings.

\section{Conclusions}

The different approaches available for studying natural orchid germination are best used in combination, so that hypotheses are based at least partly on observation of the unmanipulated system, and supplementary test in vitro used to address specific questions in more detail. The backbone technique remains the sowing in situ and all the experimental options it has to offer.

\section{Acknowledgements}

My gratitude goes to the organizers of this conference and the colleagues who instituted and supported the series of IOCC that have been very successful in stimulat- ing international collaboration on orchid conservational studies. An anonymous reviewer supplied valuable suggestions for improvement of the text, for which I am thankful.

\section{REFERENCES}

Batty AL, Dixon KW, Brundrett M, Sivasithamparam K (2001) Constraints to symbiotic germinaton of terrestrial orchid seed in a mediterranean bushland. New Phytol 152: 511-520.

Bernard N (1899) Sur la germination du Neottia nidus-avis. Comp. Rend. Hebd. Seánces Acad. Sci., Paris, 128: 1253-1255.

Brundrett MC, Scade A, Batty AL, Dixon KW, Sivasithamparam K (2003) Developmnet of in situ and ex situ seed baiting techniques to detect mycorrhizal fungi from terrestrial orchid habitats. Mycol Res 107: 1210-1220.

Chung MY, Nason JD, Chung MG (2004) Spatial genetic structure in populations of the terrestrial orchid Cephalanthera longibracteata (Orchidaceae). Amer J Bot 91: 52-57.

Corey LL, Moller-Jacobs LL, Ross AY, Zettler LW, Richardson LW (2011) Protocorms of a epiphytic orchid (Epidendrum amphistomum A. Richard) recovered in situ, and the subsequent identification of associated mycorrhiza fungi using molecular markers. This volume.

Crackles E (1975) The Monkey Orchid in Yorkshire. The naturalist 932: $25-26$.

Diez JM (2007) Hierarchical pattertns of symbiotic orchid germination linked to adult proximity and environmental gradients. J Ecol 95: 159-170.

Jacquemyn H, Brys R, Honnay O, Roldán-Ruiz I, Lievens B, Wiegand T (2011) Nonrandom spatial structuring of orchids in a hybrid zone of three Orchis species. New Phytologist doi: 10.1111/j.1469-8137-2011-03913-x.

Keel BG, Zettler LW, Kaplin BA (2011) Seed germination of Habenaria repens (Orchidaceae) in situ beyond its range, and its potential for assisted migration imposed by climate change. Castanea 76: 43-54.

Manning JC, Van Staden J (1987) The development and mobilisation of seed reserves in some African orchids. Austr J Bot 35: 343-353.

McCormick MK, Whigham DF, Sloan D, O’Malley K, Hodkinson B (2006) Orchid-fungus fidelity: a marriage meant to last? Ecology 87: 903-911.

McKendrick SL, Leake JR, Read DJ (2000) Symbiotic germination and development of myco-heterotrophic plants in nature: transfer of carbon from exomycorrhizal Salix repens and Betula pendula to the orchid Corallorhiza trifida through shared hyphal connections. New Phytol 145: 539-548.

Rasmussen H (1990) Cell differentiation and mycorrhizal infection in Dactylorhiza majalis (Orchidaceae) during germination in vitro. New Phytol 116: 137-147.

Rasmussen HN (1995) Terrestrial orchids - from seed to mycotrophic plant. Cambridge Univ Pr.

Rasmussen HN, Pedersen HÆ (2011) Cypripedium calceolus germination in situ. Seed longevity, and dormancy breakage by long incubation and cold periods. This volume.

Rasmussen HN, Whigham D (1993) Seed ecology of dust seeds in situ: a new study technique and its application in terrestrial orchids. Amer J Bot 80: 1374-1378.

Rasmussen HN, Whigham DF (1998a) The underground phase: A special challenge in studies of terrestrial orchid populations. Bot J Linn Soc 126: 49-64. 
Rasmussen HN, Whigham DF (1998b) Importance of woody debris in seed germination of Tipularia discolor (Orchidaceae). Amer J Bot 85: 829-834.

Richardson KA, Peterson RL, Currah RS (1992) Seed reserves and early symbiotic protocorm development of Platanthera hyperborea (Orchidaceae). Can J Bot 70: 291-300.

Ridley HN (1930) The dispersal of plants throughout the world. Ashford, Kent: Reeve and Co.

Swarts ND, Sinclair EA, Francis A, Dixon KW (2010) Ecological specialization in mycorrhizal symbiosis leads to rarity in an endangered orchid. Molec Ecol 19: 3236-3242.

Trapnell DW, Hamrick JL, Nason JD (2004) Three-dimenstional fine-scale genetic structure of the neotropical epiphytic orchid, Laelia rubescens. Molec Ecol 13: 1111-1118.
Whigham DF, O’Neill JP, Rasmussen HN, Caldwell BA, McCormick MK (2006) Seed longevity in terrestrial orchids potential for persistent in situ seed banks. Biol Conserv 129: 24-30.

Whigham DF, O’Neill J, McCormick M, Smith C, Rasmussen HN, Caldwell B, Daniell T (2002) Interactions between decomposing wood, mycorrhizas, and terrestrial orchid seeds and protocorms. In: P Kindlmann, JH Willems, and DF Whigham (eds), Trends and Fluctuations and Underlying Mechanisms in Terrestrial Orchid Populations. pp 117-131. Backhuys Publishers, Leiden, The Netherlands.

Willems JH (1982) Establishment and development of a population of Orchis simia Lamk. in the Netherlands, 1972 to 1981 . New Phytol 91: 757-765. 